\title{
The Use of Actuated Flexible Plates for Adaptive Shock Control Bumps
}

\author{
Edward Jinks*, Paul J.K. Bruce ${ }^{\dagger}$ and Matthew Santer ${ }^{\ddagger}$ \\ Imperial College London, $S W^{\prime}$ 2AZ, United Kingdom
}

\begin{abstract}
Adaptive shock control bumps (SCB) aim to retain the performance of static SCV and improve off-design performance caused by variations in shock position due to flowfield unsteadiness and changes in aircraft cruise conditions. An adaptive SCB requires the flexibility to deform and the stiffness to withstand the complex pressure field that is present on the upper surface of a transonic aircraft wing. This study uses a quasi-steady aerostructural solver to design adaptive SCB. Both flexible and actuated plates have been tested in a Mach 1.4 blowdown wind tunnel. The shock structure has been captured over both plates $(\mathrm{t}=\mathbf{0 . 4 \mathrm { mm }})$ using high speed Schlieren Imaging. Experimental results show that the flexible and actuated plates both have a stabilising effect on the shock, reducing the amplitude of unsteady shock motion from $30 \mathrm{~mm}$ to $20 \mathrm{~mm}$ and $10 \mathrm{~mm}$ respectively. In addition, the actuated plate enabled the shock's mean stream wise position to be varied by up to $26 \mathrm{~mm}$ for an actuator displacement of just $3 \mathrm{~mm}$. The shock holding characteristics were attributed to how changes in surface curvature caused by the cavity pressure and actuation affected the external flow structure and shock structure. The cavity pressure beneath a flexible plate is shown to be a significant design variable with the plate geometry moving from a depression to a protrusion with just 0.1 bar variation.
\end{abstract}

\section{Nomenclature}

$\Delta P_{\text {cav }}$ Pressure in cavity region

$\lambda^{*} \quad$ Flutter boundary dynamic pressure

$\nu \quad$ Poisson's ratio

$\sigma_{y} \quad$ Material yield stress

$\sigma_{v M} \quad$ von Mises stress

$C_{p} \quad$ Pressure coefficient

$h_{b} \quad$ Bump height

$\begin{array}{ll}l_{b} & \text { Bump length } \\ M_{\text {shock }} & \text { Mach number upstream of shock } \\ M_{w t} & \text { Wind tunnel Mach number } \\ \mathrm{a} & \text { Panel length } \\ \mathrm{b} & \text { Panel width } \\ \mathrm{c} & \text { Airfoil chord } \\ \mathrm{D} & \text { Flexural stiffness } \\ \mathrm{E} & \text { Young's Modulus } \\ \mathrm{q} & \text { Dynamic pressure } \\ \mathrm{t} & \text { Plate thickness }\end{array}$

\section{Introduction}

$\mathrm{T}^{\mathrm{N}}$ this paper we present an investigation of adaptive shock control bumps (SCB). Flow control techniques have been an established part of aeronautics for many decades and have ranged from early stage wing fences to highly adaptable morphing wings. Shock control bumps are one such type of flow control device which has shown promise for use on conventional supercritical wings. The large extent of favorable pressure gradient extending over the upper surface results in a large pocket of supersonic flow which is terminated by a strong near-normal shock wave, typically $M_{\text {shock }} \sim 1.4$ as shown in figure 1a. This shock wave typically oscillates over a region $\sim 3 \%$ c. Low frequency movements are due to different cruise points and changes in altitude whilst the high frequencies are due to genuine shock unsteadiness. It is this unsteadiness that bounds subsonic aircraft through over-cautious design, if the conditions surrounding the unsteadiness can be better understood then more efficient designs could be produced.

*AIAA Student Member

† Senior Lecturer in Aerodynamics, AIAA Member

${ }^{\ddagger}$ Senior Lecturer in Aerostructures, AIAA Senior Member 


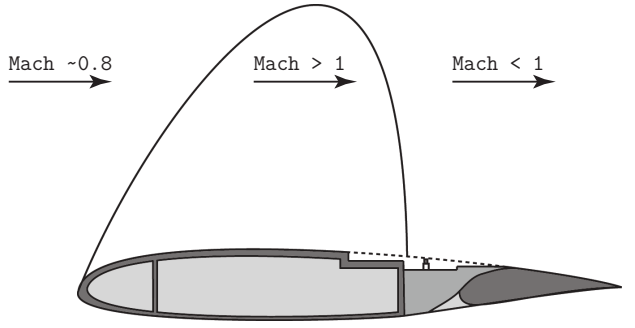

(a) Retracted SCB.

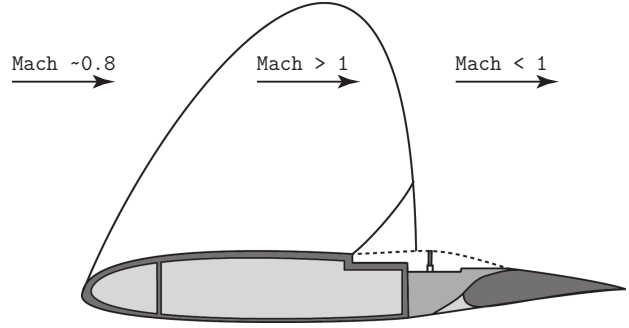

(b) Deployed SCB.

Figure 1. Typical shock structure for transonic applications, sonic line shown. ${ }^{1}$

The SCB was originally proposed as a concept by Ashill ${ }^{1}$ and in its original guise featured a flexible surface that could deform to create a bump beneath a shock. This then triggered the structure shown in figure $1 \mathrm{~b}$ whereby the initial deflection causes compressive pressure waves to form in the supersonic region. These waves can coalesce to form an oblique shock referred to as the front leg of the $\lambda$-shock, as seen infigure 2 , regions (1)-(2). This intersects with the wings main normal shock at the triple point which bounds the height of large-scale SCB effect. The drag reducing properties stem from basic shock theory that decelerating the flow through a two shock system is more efficient at maintaining total pressure than through a single normal shock. By reducing the Mach number in region (2) the strength of the normal shock between regions (2)-(3) is significantly lowered and thus the overall pressure gradient lessened.

The shock control bump shows maximum potential when applied to wings with comparatively strong shocks $\left(M_{\text {shock }} \sim 1.4\right)$ as the $\lambda$ shock structure smears the shock over a larger area. This is contrary to typical airfoil design which aims to minimize shock strength. Even in the absence of SCB some smearing of the shock occurs naturally over a flat surface due to the presence of a boundary layer with strong shocks and hence shockwave boundary layer interaction (SBLI). The role of SCB is to smear the pressure rise further to allow for stronger shocks without the detrimental separation. By increasing the area over which the shock induced pressure gradient acts, the likelihood of separation due to the stronger shock can be reduced as depicted in figure 2.

The initial pressure rise through the front leg of the $\lambda$ produced by SCB is a function of the flow deflection angle set by the angle of the leading face of the SCB. These pressure changes over the surface of the shock control bumps are very sensitive to surface geometry.
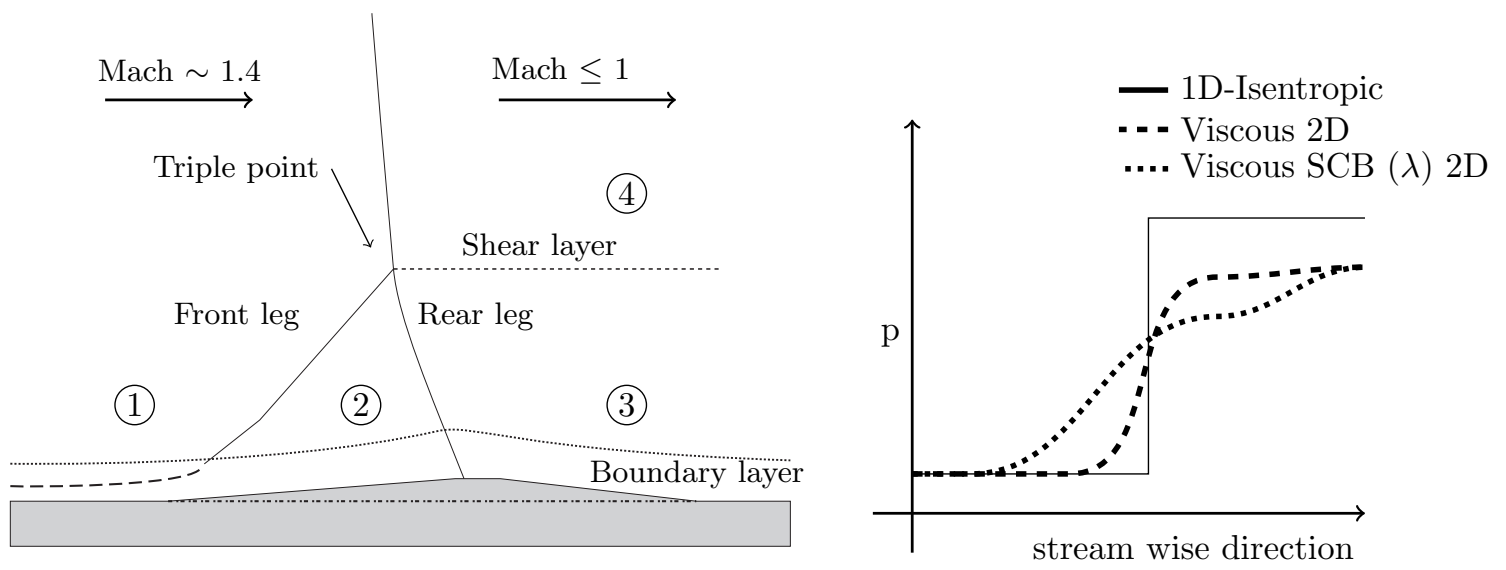

Figure 2. Typical $\lambda$-shock structure across an SCB

Despite Ashill's original concept of an adaptive SCB, the vast majority of studies that have subsequently considered SCB have focussed upon static bumps that remain present throughout an aircraft's entire envelope 
of flight. This is partly due to limitations in experiments and time resolved CFD. As a result, these studies focus purely on the optimization of aerodynamic quantities for steady cruise conditions. ${ }^{2,3}$ This approach does overlook the off-design performance of SCB including effects due to oscillations of the shock. Including the unsteadiness in the optimal design process is very computationally expensive, however multiple discrete flow conditions would provide some information regarding the versatility of the designs. Even with this discrete approach of evaluating off-design cruise conditions the intermediate aerodynamic stages would be altered due to the presence of a fixed SCB. To overcome this requires an adaptive approach.

One solution to minimize off-design penalties is to add deployment capabilities to the bumps. With this approach the off-design performance problems are removed and therefore the SCB can always be on-design by adapting to the environment. This of course is the theoretical approach however this study highlights that the design for adaptive SCB is far more complex than inserting an adaptive structure. Using active control to create typical SCB geometries through actuators is one approach ${ }^{4}$ however the pressure field surrounding the shock could be used to deform flexible plates in order to have a similar effect. This paper aims to build upon ${ }^{5}$ the aerodynamic and structural behavior of adaptive SCB and the implications involved in the deployment of these bumps. In particular the design using a quasi-static aerostructural solver and the transient response through experimentation of actuated and flexible plate SCB.

The aims of this paper are to:

- Identify the limits of a quasi-static design approach to adaptive SCB.

- Experimentally investigate the response of a flexible plate to shock loading without actuation.

- Measure the performance of an actively controlled adaptive SCB under shock loading.

\section{Adaptive SCB Design}

Moving from static fixed shape SCB to adaptive SCB necessitates a coupled approach between structural dynamics and aerodynamics. The unsteady dynamic mechanisms associated with flexible plates and shockwaves are not well understood and very few studies have looked at these in detail. This is due to the high computational cost of fluid structure interactions as well as the long time scales that occur in physical experiments.

Whilst the geometries of static two and three dimensional SCB have been developed and optimized previously ${ }^{6,7,8}$ the development of adaptive SCB has been restricted to structural models that have not been tested in a wind tunnel. ${ }^{7}$ Rhodes $^{4}$ focussed upon designing adaptive 3D structures and highlighted the difficulties in reaching suitable levels of performance from an adaptive three dimensional SCB. These were limited to static loading and the limiting factor being the achievable height whilst maintaining elastic deformation of the SCB material.

The continuous geometry of deformed beam shapes has been found to preserve the quality of the boundary layer very well. ${ }^{9}$ On an airfoil this equates to less severe pressure distribution changes along the top surface. Such geometries will be beneficial in terms of geometric and manufacturing constraints as shown in previous optimization studies. ${ }^{5}$ 
On an airfoil of chord length c, typical SCB heights range from $0.4 \% c \leqslant h_{b} \leqslant 1.04 \% c^{10,11}$ with lengths, $l_{b} \sim 0.2 \mathrm{c}$. In order to achieve such deflections the material must be flexible out of plane yet relatively stiff once deformed to maintain the geometry. The geometry of a loaded beam shape is to be replicated by a plate in two-dimensional bending to investigate $2 \mathrm{D}$ adaptive SCB.

In comparison to airfoil studies SCB tested in wind tunnels are often scaled to boundary layer height and tunnel height. Due to the local Mach number where these SCB will operate the testing conditions become particularly harsh with many pressure loads apparent. ${ }^{12}$ Typical bump sizes for testing in wind tunnels are $l_{b} \sim 200 \mathrm{~mm}$ with bump heights $\delta_{\text {crest }} \sim 6 \mathrm{~mm} .{ }^{13,14}$ The differences between designing for airfoils and wind tunnels will be highlighted in section II.B.

\section{A. Quasi-Steady Aerostructural Design Tool}

CFD analysis is carried out using the rhoCentralFoam solver within OpenFoam that has been validated previously for optimal design studies. ${ }^{5}$ A traditional D-mesh is used with 10c radius, $15 \mathrm{c}$ downstream with $y^{+} \approx 30$. This is coupled with a shell analysis completed in Abaqus ${ }^{15}$ with S4R shell elements. 13000 elements have been used which has been shown to give converged results. Figure 3 depicts the process.

The RAE 2822 supercritical airfoil is used as the baseline geometry with features typical of transonic airfoils. The curvature over the upper surface of the airfoil is very gradual with a maximum thickness of $0.121 \mathrm{c}$ occurring at $50 \% \mathrm{c}$. The stronger curvature over the rear half of the upper surface lends itself to a more stable shock position whilst maintaining attached flow.

CFD results have been compared to an experimental study on the same airfoil ${ }^{16}$ with comparable shock position and surface pressure distribution. Figure 4a shows that the model is a suitable representation of the experiment and this setup is used in the coupling. The upper and lower bounds represent the highest and lowest $C_{p}$ values, the mean value is the average across 100 iterations. This strengthens the case of the CFD setup to ensure that the correct physics are being used in the coupling process. The quasi-steady analysis shows the general features of the flexible plate without the computational expense of time-resolved simulations. The iterative design process between computations and initial experiments enables the quick evaluation of plate characteristics leaving the time resolved aspects to experimental investigation.

The aerostructural solver is comprised of the structural and aerodynamic models ${ }^{5}$ and features a flexible section between $0.4 c \leq x \leq 0.6 c$. The shock position is naturally maintained at $\sim 0.5 c$ so the pressure rise is approximately in the centre of the control region. As evaluated previously the material requires an exceptionally high yield stress to maximise displacement through elastic deformation. Al-7075-T6 has been selected and will be modelled both numerically and in experiments. A thickness of $0.4 \mathrm{~mm}$ provides enough flexibility to sustain suitable displacements. The length of the plate $l_{b}=0.2 \mathrm{c}$ is used, this length performed well in previous parametric studies. ${ }^{17}$ For dimensional analysis the airfoil chord is taken to be $1 \mathrm{~mm}$.

Through the quasi-steady coupling process in figure 3 the converged CFD pressure distribution is mapped on to the plate for FEA analysis. This produces a new geometry and the CFD solution is re-meshed and resumed. The pressure profiles in figure 4 show the bounds of variation in $C_{p}$ through 100 iterations of the coupled solver. It has been determined from the quasi-static results that the adaptive SCB should be able to withstand the forces present around the SBLI region.

\section{B. Unsteady Aeroelastic Behavior of Flexible Plates}

The flexibility of panels and pressure loading at transonic speeds is a topic of aeroelastics that was first documented in the late 1960's. In particular the effects of panel flutter reviewed by Dowell. ${ }^{18}$ The physical nature of the problem stems from pressure changes above the plate which causes a deflection of the panel. The initial amplitudes of the response are typically $\sim 0.01 \mathrm{t}$ however above the flutter boundary amplitudes grow to $\sim$ t.

The flutter boundary represents a stagnation pressure whereby the amplitude response grows sharply. It is much more likely to occur on an airfoil operating over a wide range of dynamic pressures. The onset can typically be identified to within $10 \%$ dynamic pressure for a given plate with existing empirical measures. The flutter dynamic pressure, $\lambda^{*}=\lambda_{\text {flutter }}^{*}(\mu, M, a / b)$, is calculated by applying a factor relating the flexural stiffness of the plate, the dynamic pressure and the length, equation 1. Applying the dimensions of the flexible plate flutter dynamic pressure $\lambda^{*}=1800$. This is well beyond the value for flutter onset at $M_{f r}=1.4$ which is $\lambda^{*} \approx 600$, the response is expected to be in excess of $1.6 t .{ }^{19}$ 


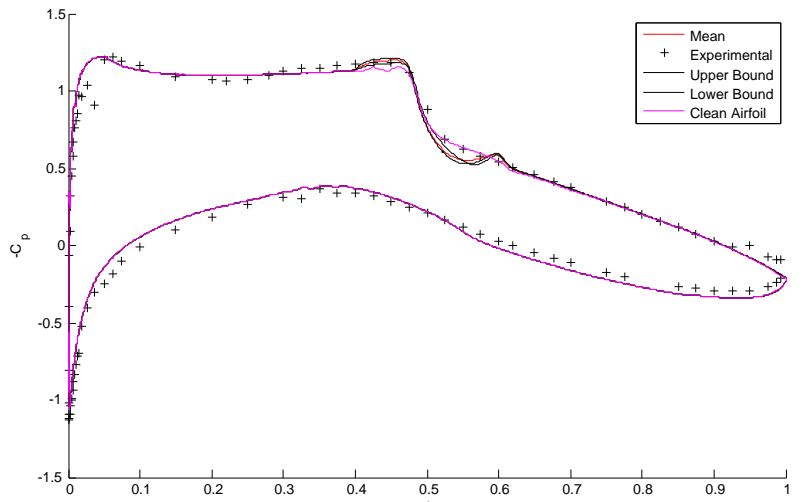

$(a)^{x / c}$

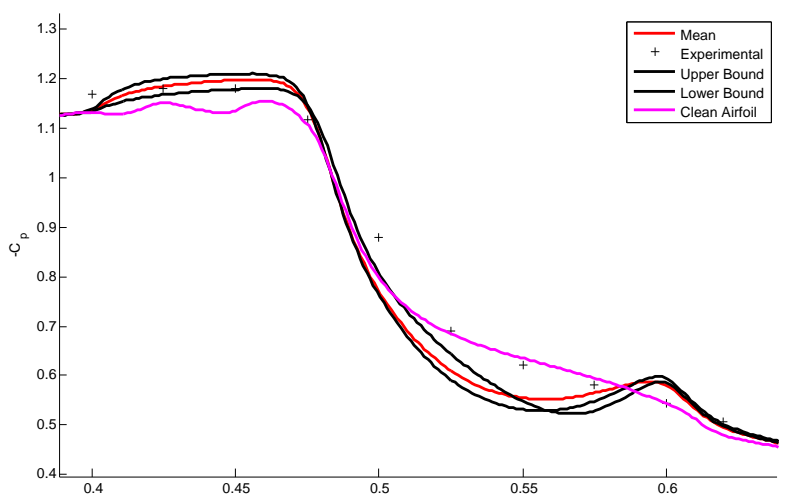

$(\mathrm{b})^{x_{6}}$

Figure 4. Pressure profile bounds and mean profile over 100 iterations of quasi-steady aerostructural solver. (a) Entire profile. (b) represents the area of interest.

$$
\begin{array}{r}
\lambda^{*}=\frac{2 q a^{2}}{D} \\
D=\frac{2 h^{3} E}{3\left(1-\nu^{2}\right)}
\end{array}
$$

The clamped ends of the plate offer a significant constraint to the problem in the static analysis and the dynamic effects via damping. Introducing the clamped constraints, the tension on the upper surface increases the effective plate stiffness. This reduces the amplitude and frequency of any oscillations of the panel due to the flow conditions. ${ }^{12}$

The two dimensional coupled solver does not take into account the spanwise variations of the plate geometry. This allows for quick analysis of varying thicknesses, lengths and cavity pressures. All are important with respect to the flutter boundary. Due to the location of SCB both on airfoils and within wind tunnels, the volume surrounding the plate is relatively small and the dynamics of the air in the cavity will play a significant role. As the shock is passed over the flexible plate, the stream wise pressure gradient is significantly affected and the plate will be subjected to a complex pressure field. This will affect the geometry and therefore the pressure field resulting in the interdependencies shown in figure 5 .

Investigating the aeroelastic behaviour of flexible plates for adaptive SCB requires two approaches: first incorporating the design into airfoils and second the study of the shock structures in the flow.

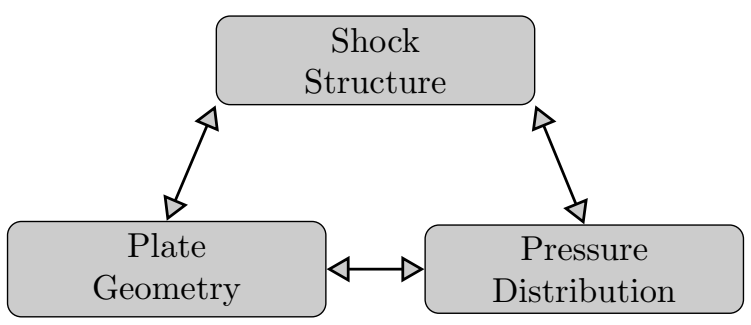

Figure 5. The interdependencies of shock structure, pressure gradient and plate geometry.

\section{Airfoil Design}

For integration into airfoil designs, the original concept must be referred to, shown in figure 1. Treating SCB as a modification to existing airfoils the adaptive SCB surface may have to be curved in its default state to maintain existing geometry. 
From previous optimization studies, ${ }^{5}$ the most significant factor affecting performance of adaptive SCB is the position of the bump relative to the shockwave. Airfoil designs usually place the shock in a region of small convex curvature to hold the shock position. Comparisons can be made between supercritical and NLF airfoils. ${ }^{20}$ The stronger shocks associated with the latter and the advances in NLF research are beneficial for adaptive SCB design.

The SCB geometry also needs to blend with the existing geometry and a smooth transition is critical to ensure that the quality of the supersonic flow is maintained as well as the deceleration of the subsonic flow on the rear of the SCB. Analysing the small scale flows through simulations is a very time consuming process and does not lend itself to optimization studies. This is why the flow physics surrounding the SCB are much more reliably and frequently captured through experimental techniques such as Schlieren and PIV. ${ }^{21}$

\section{Flat Plate Design}

Blowdown supersonic wind tunnels allow for the in-depth analysis required and focus upon the area shown in figure 6 . They are often used to simulate the flow field in the vicinity of the shock on a transonic airfoil and allow the small scale physics to be investigated. ${ }^{22}$

The flexibility requirements of adaptive SCB have meant that the dynamics of the system are very important. The dimensions are similar to those in previous panel flutter studies ${ }^{12}$ and therefore an appreciation of panel flutter has to be incorporated into the design. In the existing wind tunnel investigation the SCB are sized with respect to the incoming boundary layer height as well as overall channel height. The $\lambda$-structure is to be visible within the window pictured in figure 9 in order to evaluate performance.

Following on from the initial specification of the material in section II the plate dimensions have been selected to have an aspect ratio $\mathrm{a} / \mathrm{b}=1.33$ with an allowable thickness between 0.4 and $1 \mathrm{~mm}$. This range should minimize the likelihood of panel flutter yet allow for a significant deformation with pressure values typical of supersonic flows. The expected deformation is of the same order as the heights of the static SCB $\delta \sim 5 \mathrm{~mm}$ allowing the dynamic behavior to materialize.

Cavity pressures within the wind tunnel model illustrated

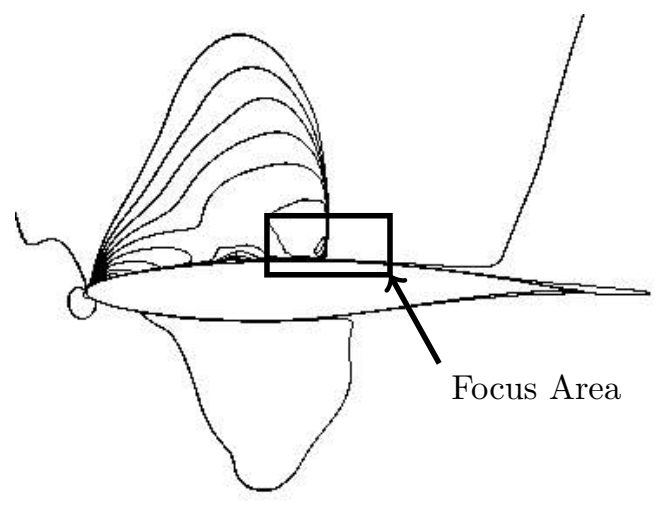
in figure 7 also need to be incorporated into the design process as the differential pressure between atmospheric and the supersonic flow is large enough to cause plastic deformation. Without sealing the cavity beneath, the plate would not return to the original geometry and would not fulfil the role of an adaptive SCB. This is visible in figure 12 where the streamwise pressure gradient is highlighted.

Sealing the cavity and incrementing cavity pressure the quasi-steady solver is used to evaluate new plate geometries. The geometries are similar to traditional SCB without the need for actuation instead relying purely upon the pressure loading. Such tests have been carried out using the aero-elastic solver previously referred to with cavity pressures ranging from 0.3 bar to 1.0 bar. Across this range, the plate geometry moves from a depression $\delta \leq 0$ to a plastically deformed plate $\delta \gg 0$. Figure 8 shows the crossover cavity pressure in which negative and positive deflections are produced with large differences in shock structure. Figures 8a-b would result

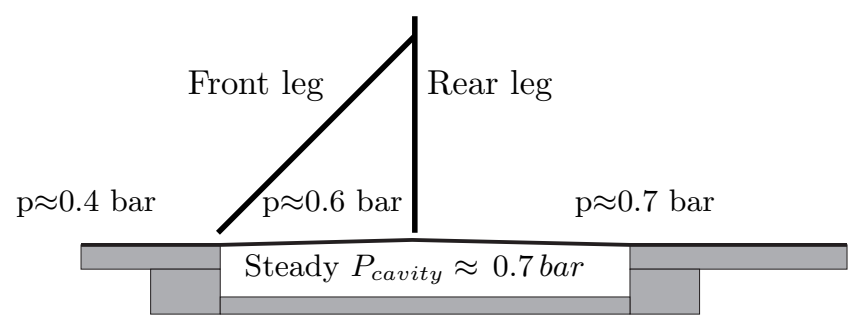
in large expansion regions $P_{\text {cavity }} \leq 0.6$ bar. Figures $8 \mathrm{e}$-f would generate the desired $\lambda$-shock structure with $P_{\text {cavity }} \leq 0.7$ bar. These values were chosen for the cavity pressure as they closely match the free stream pressures pre/post shock $\sim 0.1$ Bar above the static 
pressures present in the Mach 1.4 wind tunnel . The deflections achieved are of typical SCB heights. This highlights the importance of cavity pressures to the aerostructural problem and pushes adaptive SCB towards being passive devices. The performance of flexible plates and actuated SCB with the pressure loading is evaluated in section III.

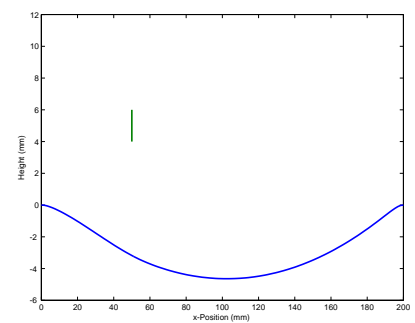

(a) $25 \%$

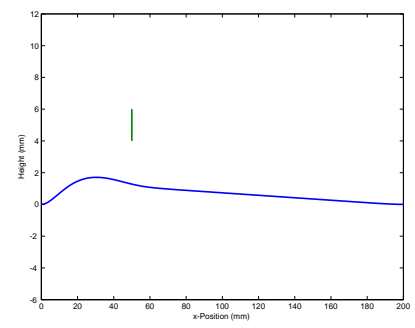

(e) $25 \%$

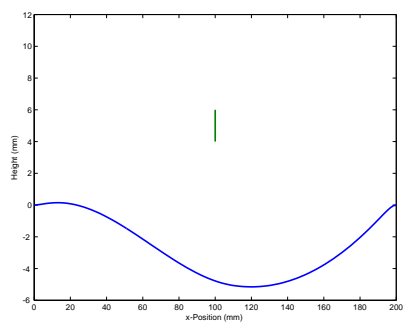

(b) $50 \%$

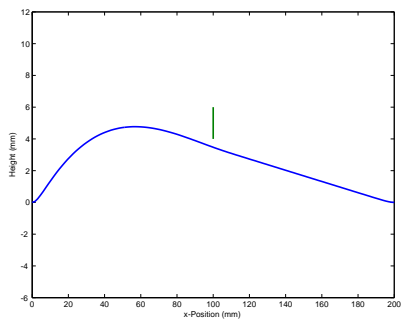

(f) $50 \%$

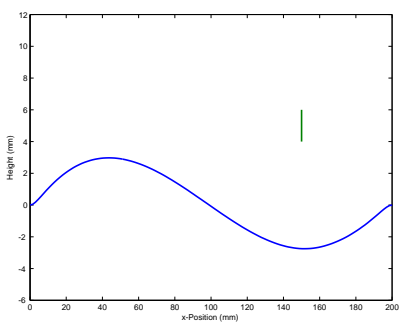

(c) $75 \%$

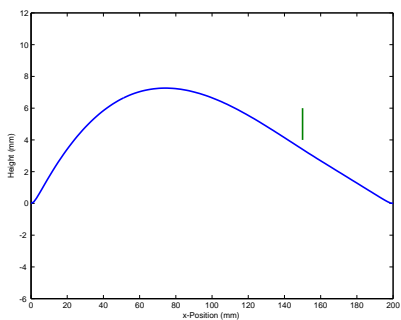

(g) $75 \%$

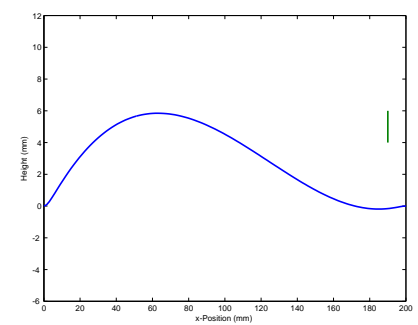

(d) $95 \%$

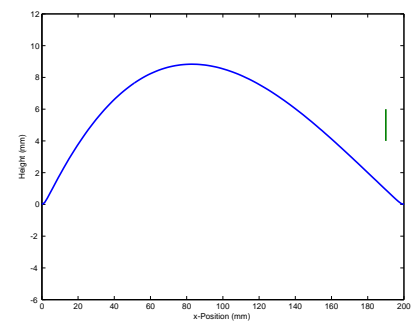

(h) $95 \%$

Figure 8. Predicted plate geometry with constant cavity pressure and shock loading. CFD shock pressure profile (over airfoil, $c=1 \mathrm{~m}$ ) mapped over flat plate, $\mathrm{l}_{\mathrm{b}}=\mathbf{2 0 0 \mathrm { mm } t}=\mathbf{0 . 4 \mathrm { mm }}$, shockwave location passing over flexible plate starting at $\mathrm{x}=0$ indicated by vertical line, see figure 6 for area of interest. Two cases with different cavity pressures (a)-(d) 0.6 Bar (e)-(h) 0.7 Bar. po $=1.29$ bar

\section{Experimental Equipment}

The Imperial College supersonic wind tunnel is a blow-down facility with run times of 60 seconds at Mach 1.4 and a working section $150 \mathrm{~mm} \times 150 \mathrm{~mm}$, figure 9 . Windows have been positioned as shown in figure 9 for Schlieren imaging. Schlieren images are obtained with an exposure time $=9 \mu \mathrm{s}$ in order to capture the fine scale unsteady structures in the flow.

The flow can be maintained at a constant pressure ratio $\Delta P_{0} \pm 1 \%$ via a LabView controller which enables shock positioning so that $x_{\text {shock }}= \pm 50 \mathrm{~mm}$. Wall pressure measurements at a resolution of $20 \mathrm{~mm}$ and frequency up to $500 \mathrm{~Hz}$ have been taken at 32 streamwise locations simultaneously using $2 \times 16$ channel pressure scanners (PSI 9116 NetScanner), with accuracies of $\pm 0.05 \%$ as in figure 9 .

High speed Schlieren images of the flow have been obtained with a Phantom v610 high speed digital camera at a frame rate of $500 \mathrm{~Hz}$ and image size of $1920 \times 1080$ pixels. This image shows the airflow in the lower half of the working section of the Mach 1.4 wind tunnel. This technique will be used to capture the large-scale motion of the shock as the bump is deployed and will provide a clear view of the transient nature of the interaction.

SCB actuation is achieved using two captive linear actuators with maximum displacements of $18 \mathrm{~mm}$ (Haydon-Kerk 25443 actuators). The small dimensions, $20 \mathrm{~mm} \times 20 \mathrm{~mm} \times 30 \mathrm{~mm}$ allowed for positioning within the shallow cavity with no air leaks.

The physical model has been manufactured and both requirements of a flat surface and clamped boundary conditions have been achieved. The quality can be seen in the profile view of the setup in figure 9. The importance of surface continuity can be seen in figures 10 and 13 where some weak Mach are seen emanating from the exit of the nozzle.

The quasi-steady structural solver has highlighted the sensitivity of plate to the pressure load; in particular with the surface continuity effects of adding a new surface to the existing geometry. The combination of 


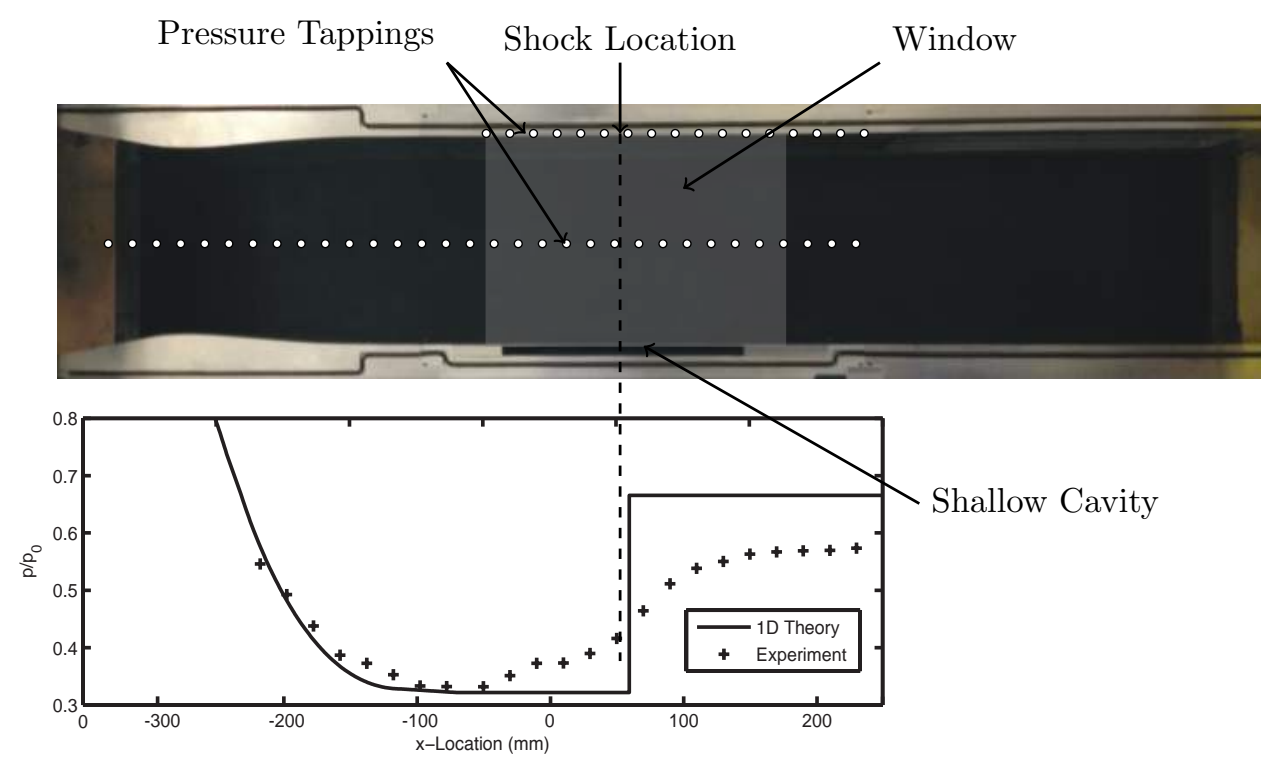

Figure 9. Mach 1.4 profile wind tunnel with adaptive SCB fitted. Experimental pressure profile with 1D theory.

these constraints and the flexibility of the plate will show the applicability of the quasi-steady solver.

\section{Adaptive SCB Performance}

The aerostructural coupling is the largest difference between current SCB design ${ }^{6,14}$ and that of an adaptive SCB. The trade-off between flexibility and stiffness is key to designing SCB to cope with the pressure loading. The results in the previous section show that adaptive SCB can be designed to interact with the flow field in a passive sense. Plates using existing aerospace materials can produce levels of deflection typical of SCB. To analyse the behavior of flexible plates and adaptive SCB physical models were produced and tested in the Supersonic Wind Tunnel at Imperial College.

\section{A. Flexible Plate Analysis}

The possibility of a flexible plate as a passive solution to the off-design performance of static SCB would potentially eliminate the need for any external energy input to the system. Without actuation the aeroelastic behavior is purely a function of shock position. Schlieren images coupled with pressure measurements hold a wealth of information regarding the dynamics of the case.

The Schlieren images in figure 10 show the changing shock structure as it passes slowly over the flexible plate. The shock position and plate surface were extracted from the video in order to analyse the response and determine the suitability of a quasi-steady solver. The images cover a physical area of $165 \mathrm{~mm} \times 90 \mathrm{~mm}$. This omits the ceiling of the tunnel at $150 \mathrm{~mm}$ and the resultant shock structure. The omission did allow for much longer sampling times that highlighted the shock holding properties of flexible plates and adaptive SCB.

The position of the shock was held on the rear surface of the SCB whilst maintaining a steady pressure ratio across the nozzle. The amount of movement is shown in figure 11 with the majority of shock positions within a standard deviation of the mean location. Whilst a frequency analysis of these results will not be undertaken the low frequency movements are clearly visible in the figure. The instantaneous geometries created by the plate can be compared to single-sided nozzles albeit with a considerably smaller area ratio and shallower gradients. The shallow gradients present over the rear surface of the plate facilitated the movement with small changes in $P_{0}$, typically $\Delta P_{0}= \pm 1 \%$ resulting in oscillations of the order $\sim 10-10 \mathrm{~mm}$ with maximum excursions of $\sim 20 \mathrm{~mm}$. This movement can be seen in figure 11 .

The comparison to a single-sided nozzle holds well in a 2D inviscid, isentropic case when analysed with 

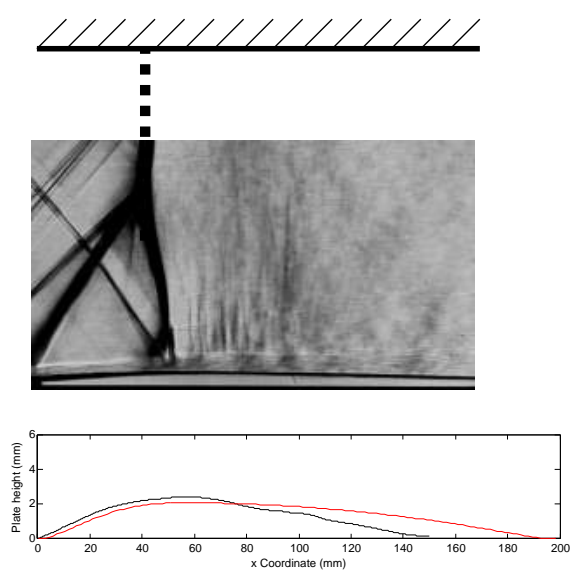
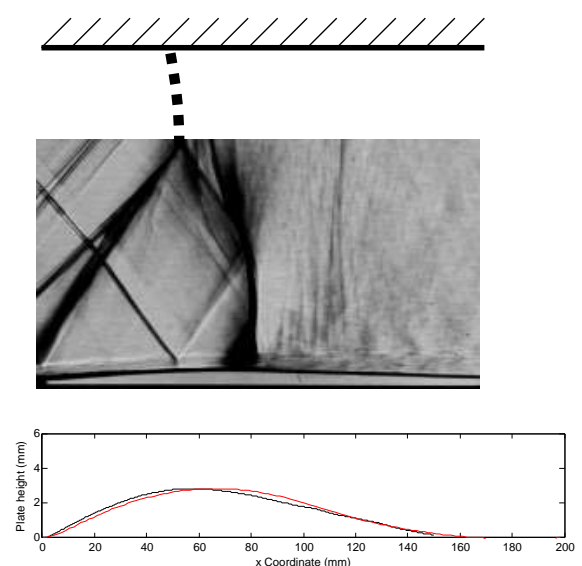
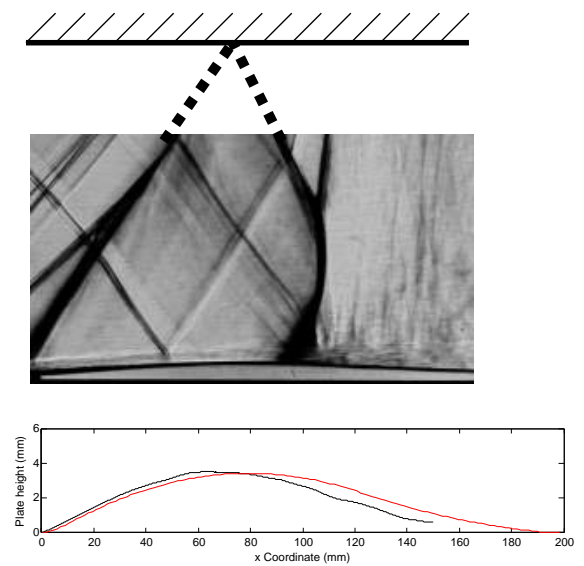

Figure 10. Shock passing over flexible plate, Al-7075-T6, t=0.4 mm, $l_{b}=200 \mathrm{~mm}$. Inferred shock structure and ceiling added to Schlieren images. Vertical scale on graphs amplified $5 \times$. Streamwise scale covers 150 mm. Experimental Results (-), FEA results (-) cover entire $200 \mathrm{~mm}$ plate.

oblique and normal shock relations. This however does not capture the true aerodynamic effects due to the fluctuating upstream pressure nor 3D nature of SBLI and the effect of the cavity. The importance of the pressure in the cavity was made apparent in the quasi-steady analysis however the motion of the shock alters this steady state pressure.

The position of the shockwave and the pressure distribution it possesses drives the pressure inside the cavity which in turn determines the geometry of the plate and therefore effects the shock structure. The illustration in figure 12 discretely highlights the differences in shock structures for each loading case.

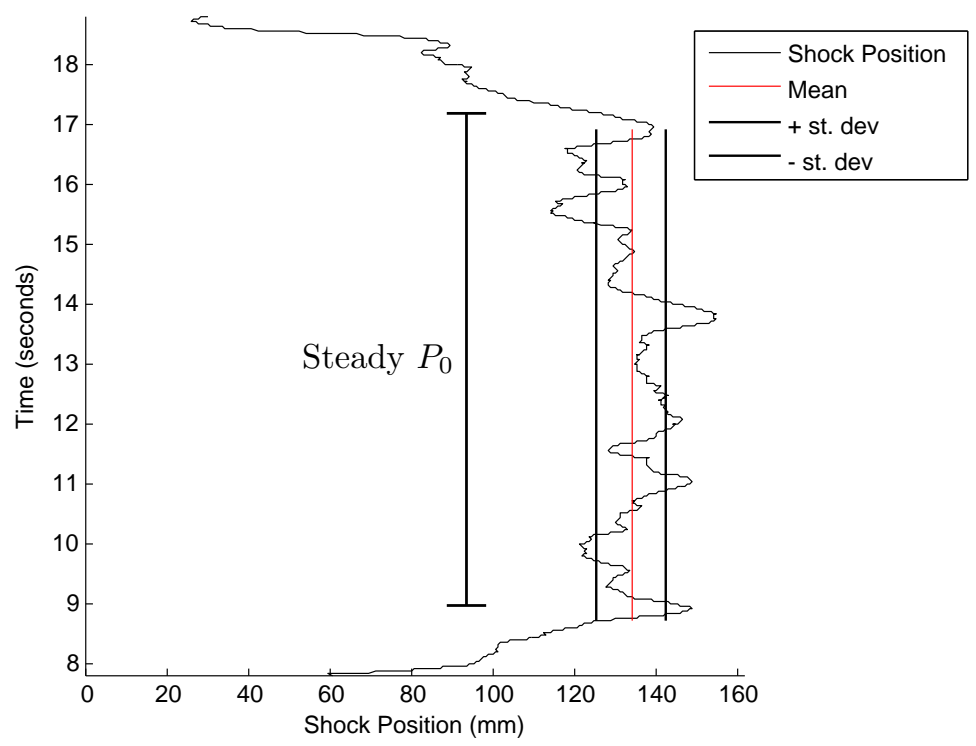

Figure 11. Shock position vs time, low-pass band filter, flexible plate, Al-7075-T6, t $=0.4 \mathrm{~mm}, 1=200 \mathrm{~mm}$, $\mathrm{b}=150 \mathrm{~mm} . \mathrm{x}$ position is represents distance along plate.

As the shock structure passed over the flexible plate the pressure gradient across the shock caused a surface deflection as predicted, figure 10. Differences between the wind tunnel measurements and the predicted shapes from the quasi-static analysis are thought to be due to variation in cavity pressure. The assumption of a constant pressure beneath the plate did not hold. This was possibly due to an air leak allowing air at atmospheric conditions to enter the shallow cavity seen in figure 9 . This raised cavity pressure and altered the pressure difference between cavity and the $\lambda$-shock region as well as the cavity and subsonic region.

The pressure within the cavity was measured at two locations, $50 \mathrm{~mm}$ and $150 \mathrm{~mm}$ from the front of 
the bump. The difference in pressure measured at two locations in the cavity is $\Delta P_{\text {cavity }} \sim 4 \%$ shown in figure 12. The measurements were $50 \mathrm{~mm}$ and $150 \mathrm{~mm}$ from the start of the plate. One of the assumptions in the flexible coupled solver was that the cavity pressure would be constant as the mass flow rates around the edges of the plate were judged to be very small. The deformation of the rear of the plate, figure 10, highlights the significant effect of cavity pressures in the differences between FEA and Schlieren plate displacement. The agreement between the latter two cases, with predominantly supersonic regions on the top surface is good. This suggests that the precise values of the cavity pressure are further complicated by the subsonic region and the movement of air caused by the pressure gradients. Any leakage of flow around the plate is highly three dimensional and may have a large effect upon the very flexible structure.

In the next section a constraint will be added to increase the stiffness and identify if active control is worthwhile.
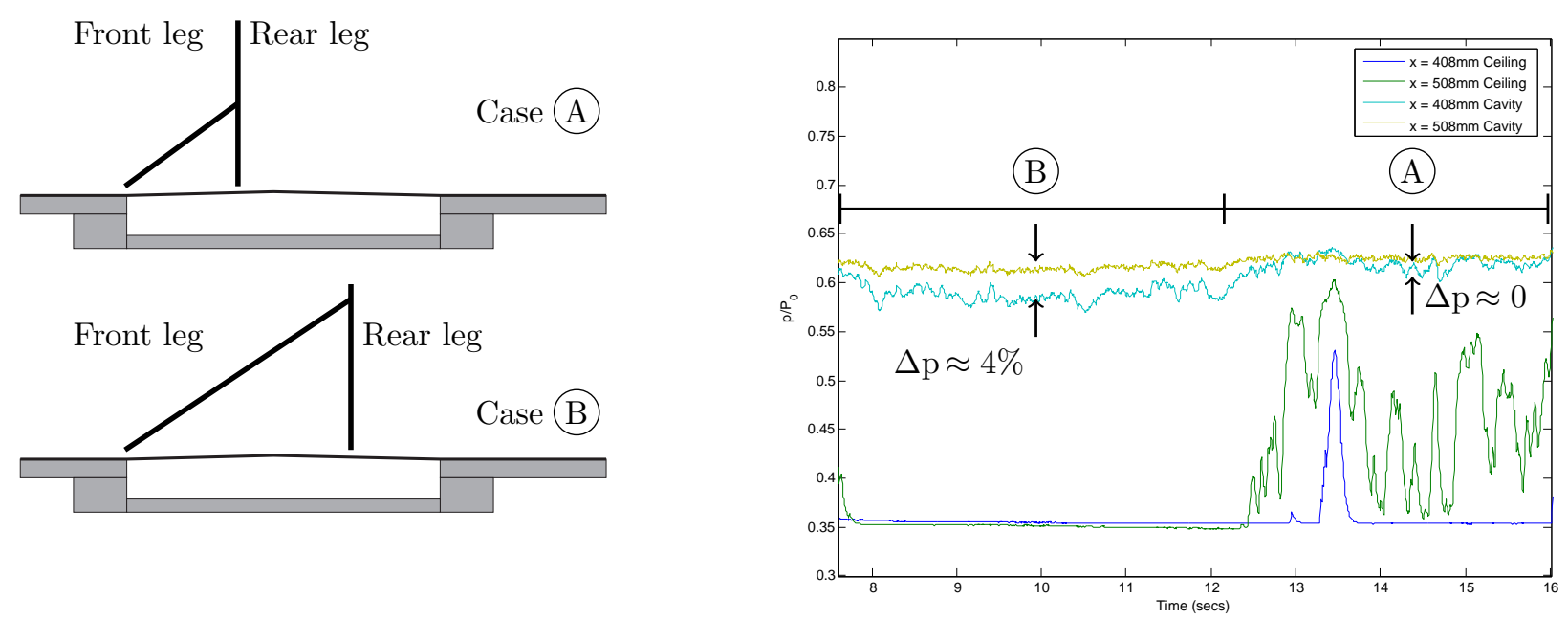

Figure 12. Cavity pressures comparison with ceiling mounted pressures (located on the surface opposite the plate, subject to full strength Mach 1.4 normal shock. Cases A and B represent the shock on the front and rear surface respectively.

\section{B. Actuated SCB Analysis}

The actuated SCB tested here builds upon the flexible plate studies shown previously. The addition of actuation has many implications to both the structural and aerodynamic aspects of the plate. Structurally, the added constraint stiffens the system reducing the effect of the pressure loading courtesy of the shock creating new geometries which affect the aerodynamics of the plate. The location of the actuation was chosen to be $0.5 l_{b}$. This allowed for maximum deflection whilst remaining within the elastic limits at the clamped ends. This geometry also performs well as a static SCB.

The added constraint effectively halves the length of the SCB and the curvature of the plate increases. The difference in curvature has a significant effect on the characteristics of SCB. The holding of a shock on a convex surface is made possible through the change in area ratios. The change in these gradients at the surface mean that a shock can be held on the rear diverging section with greater stability than in the flexible plate case. The test case here was not deformed from a flat plate due to the loading on the fixture which caused a slight reduction in length of the control region and a slight initial curvature.

In between the $\lambda$ legs there is a significant turning of the flow which can be seen by the curvature of the rear leg in figure 13a. This is a result of the expansion waves generated over the crest interacting with the rear leg and weakening it.

Studies have identified the rear portion of the shock as a critical region to focus upon, as the subsonic flow turns back to the stream wise direction. ${ }^{14}$ Although no quantitative data can be obtained from the Schlieren images regarding the quality of the flow the boundary layer is somewhat visible in both of the images. In the actuated case the flow is reaccelerated after the weakened rear leg and this normal shockwave grows and shrinks in size but does not extend beyond the triple point. The boundary layer is significantly thickened due to the presence of the shockwave. From rear leg there is no $\lambda$ in figure 13a however the rear leg splits into a $\lambda$-shock in figure 13b suggesting separation. 
Figure 14 provides the shock positions surrounding actuation. The actuation was activated for 3 seconds in order to identify if the shock returned to its original position. The total deflection was $3 \mathrm{~mm}$ with the full actuation completed with 0.3 seconds. By raising the control point the mean shock positions changed by $26.1 \mathrm{~mm}$. The movement of the shock with the actuation off was contained within $19.5 \mathrm{~mm}$ with a standard deviation of $3.6 \mathrm{~mm}$. With the actuation point raised the shock movement was between $17.2 \mathrm{~mm}$, standard deviation of $3.4 \mathrm{~mm}$. The effect of cavity pressure is much lower than in the flexible plate case as the height of the plate deflection was primarily controlled through the actuation points rather than the pressure difference. The actuation had the effect of reducing the gradient over the rear half of the SCB which allowed an easier passage for the shock to progress downstream.

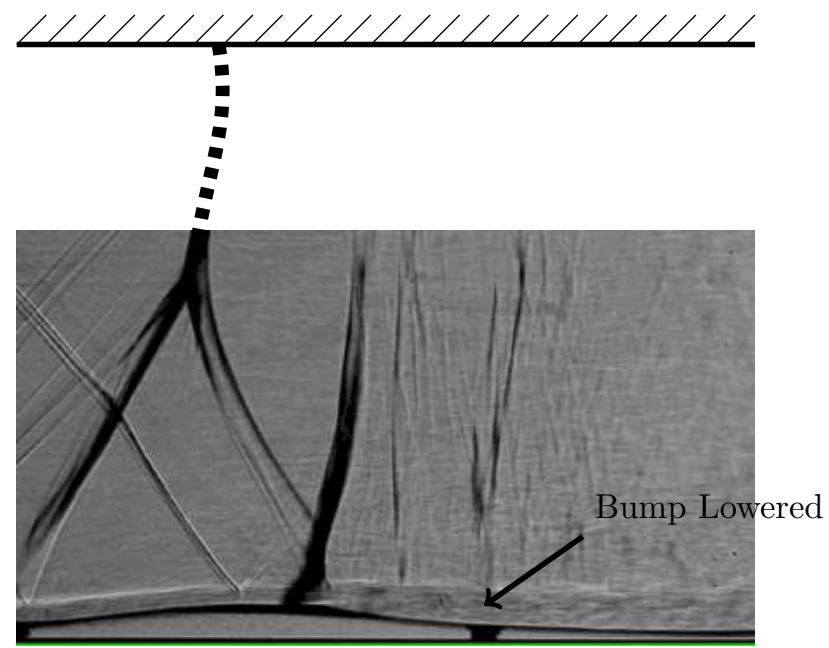

(a)

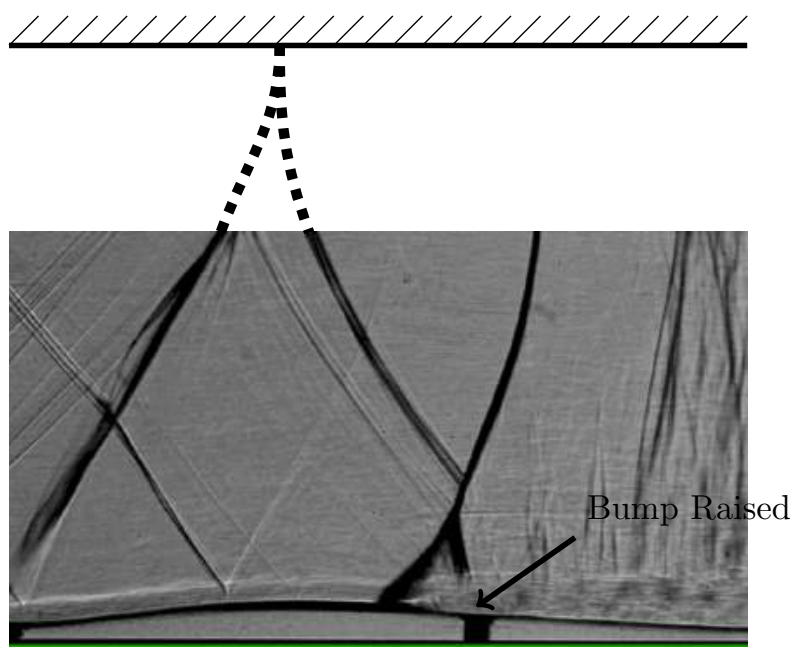

(b)

Figure 13. Shock structure through bump deployment and retraction, Al-7075-T6, t=0.4 $\mathrm{mm}, 1=200 \mathrm{~mm}$, $\mathrm{b}=150 \mathrm{~mm}$, actuation point $=100 \mathrm{~mm}$. Inferred shock structure and ceiling added to Schlieren images. Images cover $150 \mathrm{~mm}$

\section{Comparisons between flexible plates and adaptive SCB}

Both flexible and actuated plates have shown the capability of producing the desired $\lambda$-shock structure through surface deflections. The flow fields produced as a result of the changing geometry are of great interest in terms of small and large scale structures. Two important results that have come to light are the ability of actuation to stabilise the shockwave; and that cavity pressure is a significant design variable.

\section{Shock structure and stability}

The unsteadiness of the shockwave is currently something that is accommodated within airfoil and inlet design and cannot be avoided. In the parallel working section of the wind tunnel the shockwave is very unstable with the effective geometry/area being controlled by boundary layer growth. Small changes in the pressure ratio can result in large scale movements, typically these movements are $\sim 60 \mathrm{~mm}$ with the current setup of the tunnel.

The two images in figure 17 are both representative of the shock structures which SCB are attempting to achieve. The large $\lambda$-shock structure is visible in both the actuated and flexible plate cases however there are differences which will be discussed here. The front leg angles determined by the incoming Mach number, $\mathrm{M}=\sim 1.4$, and the deflection angle are $56.6^{\circ}$ and $60.3^{\circ}$ for the flexible and actuated plates respectively. The calculated deflection angles (2D oblique shock theory) are $6.8^{\circ}$ and $8.1^{\circ}$ which are reasonably close to the maximum deflection for an attached shock $\left(9.411^{\circ}\right)$. The resulting Mach numbers will therefore only just remain supersonic, $1.05 \leq M \leq 1.1$. These values are for comparison only as Schlieren imaging smears 


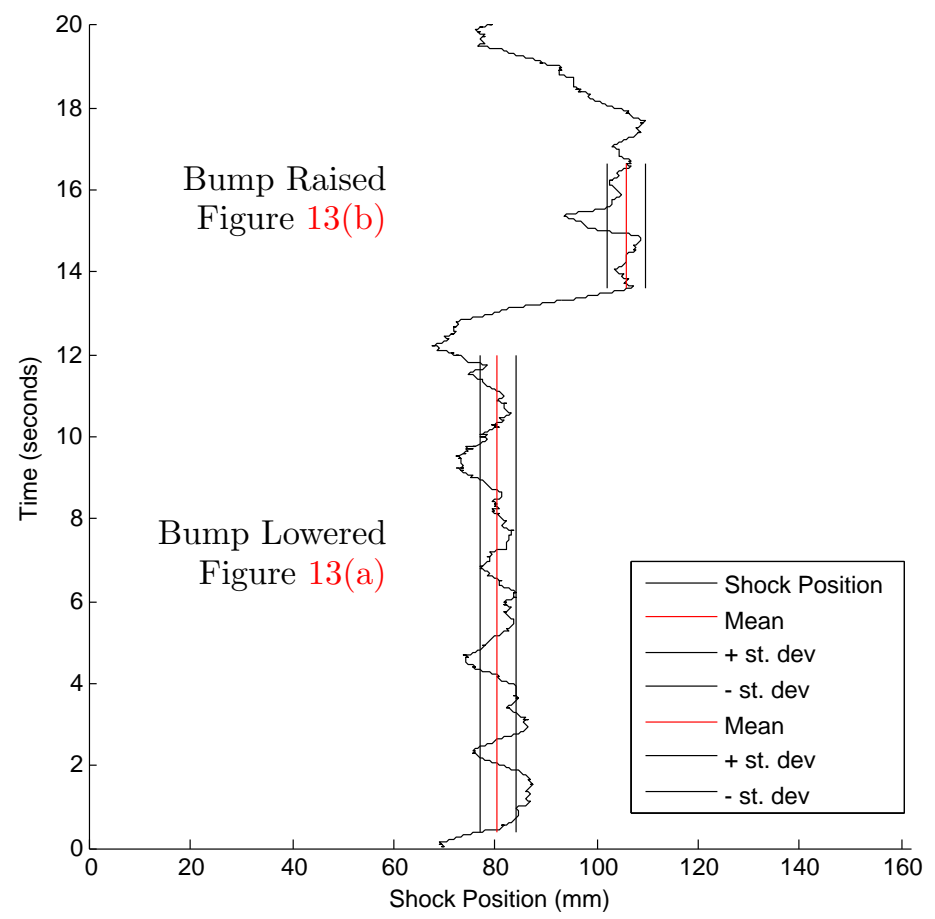

Figure 14. Shock position vs time, bump deployment and retraction.
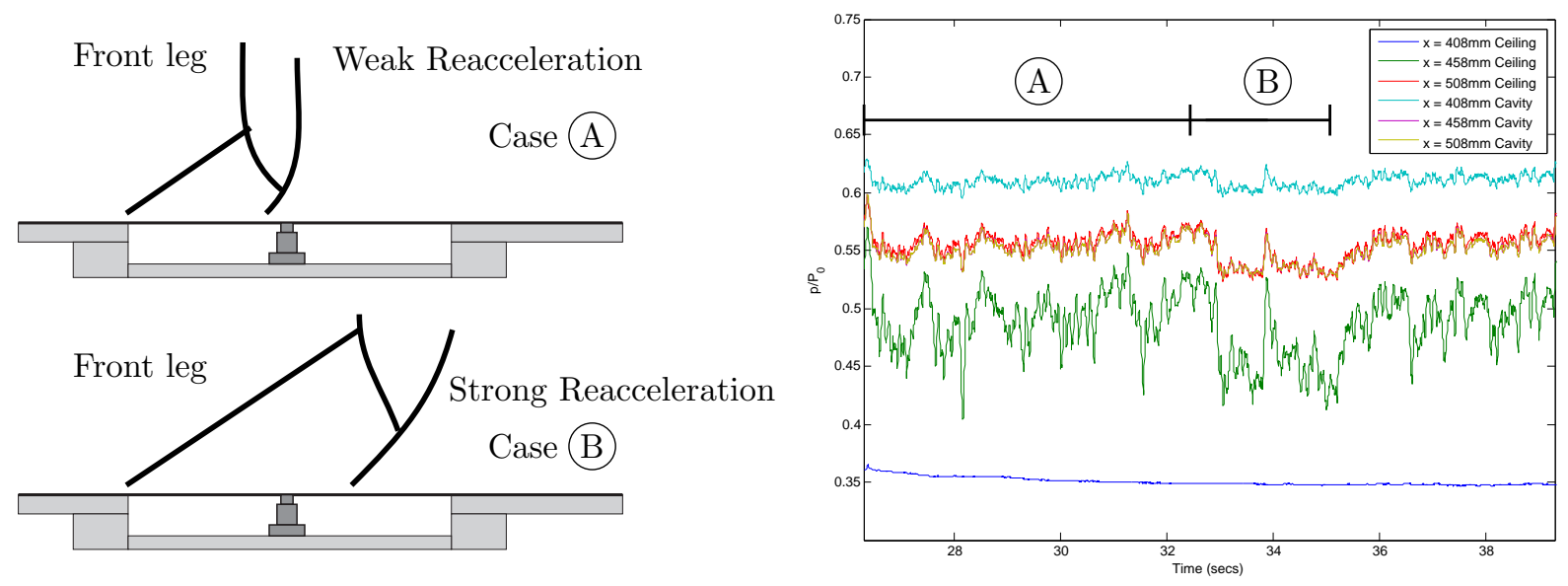

Figure 15. Cavity pressures comparison with ceiling mounted pressures (located on the surface opposite the plate, subject to full strength Mach 1.4 normal shock. Cases A and B represent control off and on respectively.

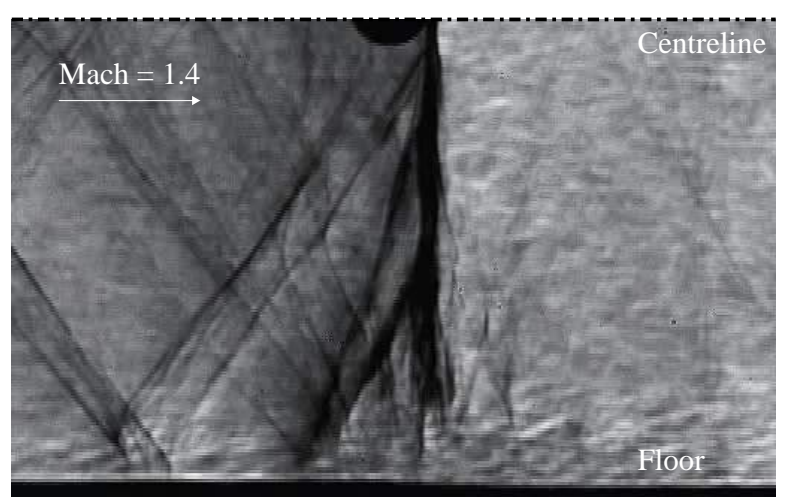

Figure 16. Baseline flow without SCB. mach 1.4 flow with normal shock wave on the rigid tunnel wall. 
the spanwise variations. This leads to a significantly weaker rear leg shock which reduces the likelihood of post-shock separation. Achieving a weak normal shock is one of the targets for SCB however it does not guarantee zero separation.

The rear legs hold the key differences between the flow fields. The actuated plate exhibits a curved rear leg attributed to the increased curvature of the plate. The effects of continuously changing deflection angles weaken which has been identified previously. ${ }^{22}$ The rear leg of the actuated case shown in figure 17 does not decelerate the flow to subsonic conditions immediately. Starting after the crest of the actuated SCB, the main normal shock does not extend beyond the triple point height suggesting a definitive secondary shock structure below the inferred structure in figure 17. The rear leg of the flexible plate case is more akin to the shock structure typically seen on a flat plate seen in figure 16 which is much more perpendicular to the wall. There is evidence of a slight bow in the rear leg which highlights the curvature of the flow field as it passes over the crest of the bump. This effect is less pronounced than the actuated case which exhibits much higher curvature. It is also apparent that the curvature of the flexible plate is due to the pressure loading both from above and beneath plate. As the cavity pressure is a function of shock position it is a major factor in the resulting geometries of the plate.

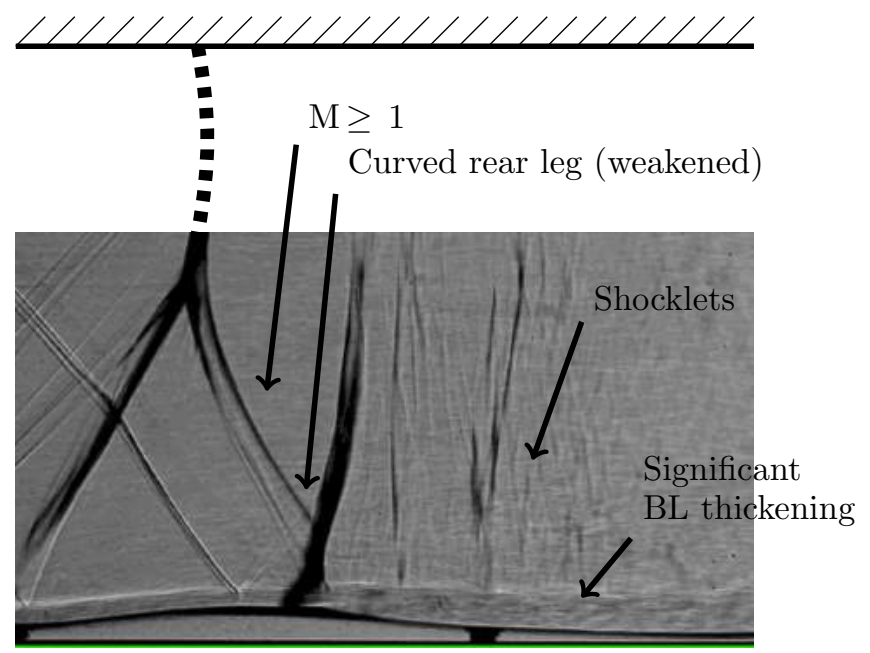

(a)

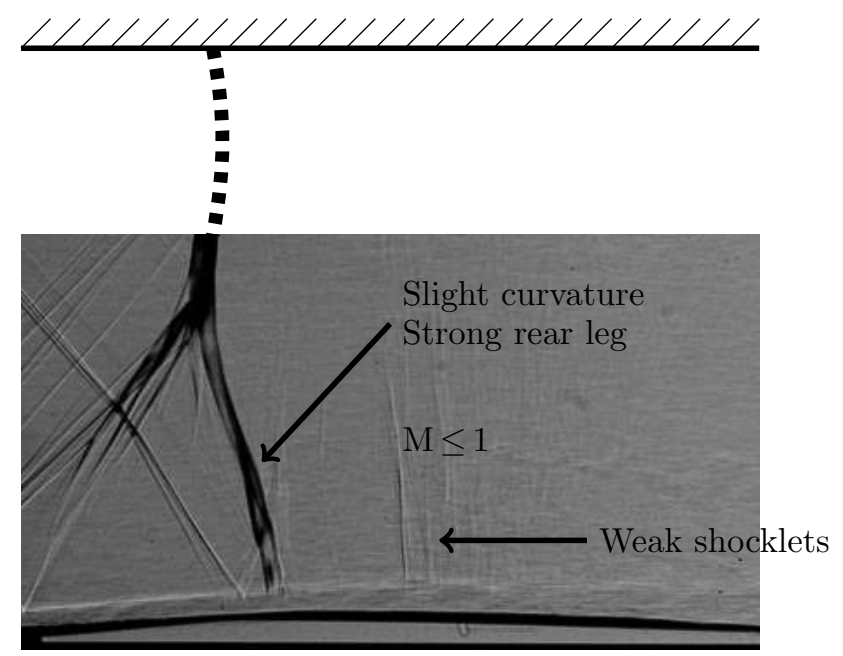

(b)

Figure 17. Comparing shock structures over actuated and flexible plates. $\mathrm{t}=0.4 \mathrm{~mm}, \mathrm{l}_{\mathrm{b}}=200 \mathrm{~mm}$

\section{Effect of cavity pressure}

The cavity pressure has a much stronger effect in the flexible case than in the actuated case due to the greater flexibility. The constraint on the actuated SCB reduces the maximum deflection and although stress levels are increased at the point of actuation. This acts as a restraint if the cavity pressure is higher than the freestream. The stress levels in the FEA at this point show $\sigma_{v M} \approx 360 \mathrm{MPa}$. This is far below the yield stress criteria of $\sigma_{y}=525 \mathrm{MPa}$. This is been confirmed in the physical model where the plate returned to a flat state when all loading was removed.

Even with the precise machine tolerances, the upper and lower surfaces of the plate were not sealed. This meant the high pressure post-shock fluid would enter the cavity which would then be sucked up by the low pressure supersonic fluid creating a cycle. Similarities can be drawn to perforated cavity studies ${ }^{23}$ which extensively studied the effects of such a cycle but without the curvature of the plate or the spanwise restrictions. Whilst the pressures in the cavity are driven by the position of the shockwave, the precise influence the pressure gradients is difficult to determine. The influence of any leakage flow upon the free stream is expected to be small based upon mass flow rates.

The results now point to a cycle which includes cavity pressure as an influential factor. This adds another factor to the original coupling diagram. The final interdependencies are presented in figure 18. The findings suggest that variable cavity pressure is an important design variable should flexible plates be used for passive 
shock control. The pressure inside the cavity would be maintained so that it remains above the static pressure of the incoming flow and below that of the post shock pressure. Further work is required to evaluate the optimal rear shapes of the cavity. An optimal design may use both cavity pressures and actuation to reached the required geometry.

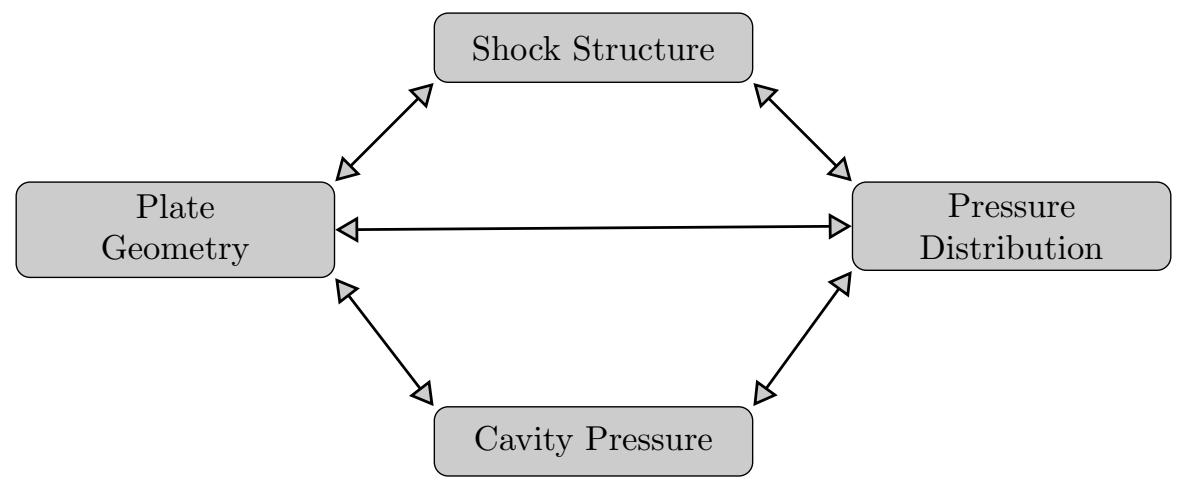

Figure 18. The interdependencies of shock structure, pressure gradient and plate geometry.

\section{Conclusion}

The investigation into the use of flexible plates for adaptive SCB has proved successful. With careful design the pressure field surrounding the shock can be utilized to create plate geometries suitable for shock control. The effects of large scale destructive panel flutter were not observed in the experimental tests however there were small oscillations due to the small variations in free stream pressure. This suggests that the quasisteady model is suitable for design purposes if the correct cavity pressure is used. The leakages between the upper and lower surfaces can cause breathing effects. These cause the results to differ from the quasisteady predictions. Maintaining a constant cavity pressure of $\sim 0.7$ Bar resulted in appropriate geometries for SCB. Varying cavity pressures would open up new possibilities in terms of achievable geometries and the combination with actuators increases this further.

Actuated SCB have shown that it is possible to control the position of the shock with a definitive change in mean shock position of $26 \mathrm{~mm}$. This was achieved with just $3 \mathrm{~mm}$ actuator displacement. The unsteady shock oscillations were reduced from amplitudes of $30 \mathrm{~mm}$ in the parallel duct to $\sim 20 \mathrm{~mm}$ with the flexible plate and $\sim 10 \mathrm{~mm}$ with actuation all dependent upon curvature. Through careful positioning of the actuation and management of the curvature on the rear slope of the SCB the range of movement could be increased. This is equivalent to changing the curvature on the top surface of the airfoil, varying stages of deployment would correspond to different shock positions. The increased curvature in the region also increased stability of the shockwave which points towards SCB being used as shock holding devices where large-scale shock movement is undesirable such as engine inlets. Such applications require the preservation of total pressure and as such the flow quality needs to be very high with minimal increases in boundary layer height. A smaller bump may therefore be desirable however the triple point height must be maximized. This would also have the effect of increasing the curvature present for a given deflection increasing shock stability.

The addition of actuation increases the curvature of the plate which significantly alters the shock structure present in figure 17. In particular the strength of the rear leg in figure 17a where it accelerates much more due to the higher levels of curvature.

The results presented here have focussed upon one particular plate thickness, $0.4 \mathrm{~mm}$. To investigate further the characteristics of flexible or actuated SCB such as SCB stiffness, tests will be performed with different thicknesses. This is to change the plate response due to the consistent Mach 1.4 shock pressure loading. Varying the stiffness would also allow for new geometries that would focus upon the shape of rear half of the SCB. This is critical to avoid significant boundary layer thickening or separation. 


\section{References}

${ }^{1}$ Ashill, P., Fulker, J. L., and Shires, A., "A Novel Technique for Controllling Shock Strength of Laminar-Flow Aerofoil Sections," First European Forum on Laminar Flow Technology, 1992, pp. 175-183.

${ }^{2}$ Yagiz, B., Kandil, O., and Pehlivanoglu, Y. V., "Drag minimization using active and passive flow control techniques," Aerospace Science and Technology, Vol. 17, No. 1, March 2012, pp. 21-31.

${ }^{3}$ Lee, D., Bugeda, G., Periaux, J., and Onate, E., "Robust active shock control bump design optimisation using hybrid parallel MOGA," Computers \& Fluids, March 2012.

${ }^{4}$ Rhodes, O. and Santer, M., "Aeroelastic Optimization of a Morphing 2D Shock Control Bump," 53rd AIAA/ASME/ASCE/AHS/ASC Structures, Structural Dynamics and Materials Conference 20th AI 23 - 26 April 2012, Honolulu, Hawaii AIAA 2012-1440, 2012, pp. 1-17.

-5Jinks, E., Bruce, P. J., and Santer, M., "Adaptive Shock Control Bumps," 52nd Aerospace Sciences Meeting, 2014.

${ }^{6}$ Klemens, N., Lutz, T., Krämer, E., Colliss, S. P., and Babinsky, H., "Shock Control Bump Robustness Enhancement," 50th AIAA Aerospace Sciences Meeting including the New Horizons Forum and Aerospace Exposition 09 - 12 January 2012, Nashville, Tennessee, No. January, 2012.

${ }^{7}$ Kutzbach, M., Lutz, T., and Wagner, S., "Investigations on Shock Control Bumps for Infinite Swept Wings," 2nd AIAA Flow Control Conference, June 27-July 1, 2004, Portland, OR, 2004, pp. 1-10.

${ }^{8}$ Wong, W., Qin, N., Sellars, N., Holden, H., and Babinsky, H., "A combined experimental and numerical study of flow structures over three-dimensional shock control bumps," Aerospace Science and Technology, Vol. 12, No. 6, Sept. 2008, pp. $436-447$.

${ }^{9}$ Sommerer, A., Th., L., and Wagner, S., "Design of Adaptive transonic Airfoils by Means of Numerical Optimization," Proceedings ECCOMAS 2000: European Congress on Computational Methods in Applied Sciences and Engineering, 2000, p. 379.

${ }^{10}$ Pätzold, M., "Numerical Optimization of Finite Shock Control Bumps," 44th Aerospace Sciences Meeting and Exhibit, Reno, Nevada, 2006, pp. 1-13.

${ }^{11}$ Lee, D. S., Periaux, J., Onate, E., Gonzalez, L. F., and Qin, N., "Active Transonic Aerofoil Design Optimization Using Robust Multiobjective Evolutionary Algorithms," Journal of Aircraft, Vol. 48, No. 3, May 2011, pp. 1084-1094.

12 Dowell, E., "Nonlinear oscillations of a fluttering plate," 3rd and 4th Aerospace Sciences Meeting, Vol. 4, No. 7, Jan. 1966.

${ }^{13}$ Ogawa, H., Babinsky, H., Pï $₫ \frac{1}{2}$ tzold, M., and Lutz, T., "Shock-Wave/Boundary-Layer Interaction Control Using ThreeDimensional Bumps for Transonic Wings," AIAA Journal, Vol. 46, No. 6, June 2008, pp. 1442-1452.

${ }^{14}$ Colliss, S. P., Babinsky, H., Nübler, K., and Lutz, T., "Vortical structures on three-dimensional shock control bumps," 51st AIAA Aerospace Sciences Meeting including the New Horizons Forum and Aerospace Exposition, No. January, AIAA, Grapevine (Dallas/Ft. Worth Region), Texas, 2013, pp. 1-15.

15 "Abaqus CAE v6.10 DassaultSystèmes," 2007.

${ }^{16}$ Cook, P., McDonald, M., and Firmin, M., "Aerofoil RAE 2822 - Pressure Distributions, and Boundary Layer and Wake Measurements," Experimental Data Base for Computer Program Assessment, AGARD Report AR 138., 1979.

${ }^{17}$ Stanewsky, E., Délery, J., Fulker, J. L., and de Matteis, P., Drag Reduciton by Shock and Boundary Layer Control: Results of the Project EUROSHOCK II, Springer, 2002.

${ }^{18}$ Dowell, E. H. P. U., "Panel Flutter - NASA SP-8004," Tech. Rep. June, 1972.

${ }^{19}$ Dowell, E. H. P. U., "Panel Flutter: A Review of the Aeroelastic Stability of Plates and Shells," AIAA Journal, Vol. 8, No. 3, 1970, pp. 385-399.

${ }^{20}$ Green, J. E., "Laminar Flow Control, Back to the Future ?" 38th Fluid Dynamics Conference and Exhibit, No. June, 2008, pp. 1-23.

${ }^{21}$ Bruce, P. J. K. and Babinsky, H., "An experimental study into the flow physics of three-dimensional shock control bumps," Journal of Aircraft, Vol. 49, No. 5, 2012, pp. 1222-1233.

${ }^{22}$ Bruce, P. J. K. and Colliss, S. P., "Review of research into shock control bumps," Shock Waves, Oct. 2014.

${ }^{23}$ Stanewsky, E., Délery, J., Fulker, J. L., and Geissler, W., Euroshock - Drag Reduction by Passive Shock Control: Results of the project EUROSHOCK, Friedrich Vieweg \& Sohn Verlagsgesellschaft mbH, 1997. 